\title{
The new lattice action and spectrum of the light and medium-mass nuclei
}

Ning Li*

Facility for Rare Isotope Beams and Department of Physics and Astronomy, Michigan State University, MI 48824, USA

E-mail: lini@nscl.msu.edu

\section{Serdar Elhatisari}

Helmholtz-Institut für Strahlen- und Kernphysik and Bethe Center for Theoretical Physics, Universität Bonn, D-53115 Bonn, Germany

Department of Physics, Karamanoglu Mehmetbey University, Karaman 70100, Turkey

E-mail: selhatisari@gmail.com

\section{Evgeny Epelbaum}

Institut für Theoretische Physik II, Ruhr-Universität Bochum, D-44870 Bochum, Germany

E-mail: evgeny. epelbaumeruhr-uni-bochum.de

\section{Dean Lee}

Facility for Rare Isotope Beams and Department of Physics and Astronomy, Michigan State University, MI 48824, USA

Department of Physics, North Carolina State University, Raleigh, NC 27695, USA

E-mail: leedenscl.msu.edu

\section{Bing-Nan Lu}

Facility for Rare Isotope Beams and Department of Physics and Astronomy, Michigan State University, MI 48824, USA

E-mail: lub@nscl.msu.edu

\section{Ulf-G. Meißner}

Helmholtz-Institut für Strahlen- und Kernphysik and Bethe Center for Theoretical Physics, Universität Bonn, D-53115 Bonn, Germany

Institute for Advanced Simulation, Institut für Kernphysik, and Jülich Center for Hadron Physics, Forschungszentrum Jülich, D-52425 Jülich, Germany JARA - High Performance Computing, Forschungszentrum Jülich, D-52425 Jülich, Germany E-mail: meissnerehiskp.uni-bonn. de

We present a new lattice formulation of chiral nuclear forces with a simpler decomposition into spin channels. With these interactions the process of fitting to the empirical scattering phase shifts is simplified, and the resulting lattice phase shifts are more accurate than in previous studies. We also present some preliminary results of spectrum of the light and medium-mass nuclei. Our results provide a pathway to $a b$ initio lattice calculations of nuclear structure, reactions, and thermodynamics with accurate and systematic control over the chiral nucleon-nucleon force.

The 36th Annual International Symposium on Lattice Field Theory - LATTICE2018

22-28 July, 2018

Michigan State University, East Lansing, Michigan, USA.

${ }^{*}$ Speaker. 


\section{Introduction}

Chiral effective field theory (EFT) organizes the interactions of nucleons in powers of momenta and factors of the pion mass near the chiral limit where the light quarks are massless. Nuclear lattice simulations using chiral EFT have been used in recent years to describe the structure and scattering of atomic nuclei $[1,2,3,4,5,6]$. However, the treatment of nuclear forces at higher orders in the chiral EFT expansion is more difficult on the lattice due to the breaking of rotational invariance produced by nonzero lattice spacing [7,8]. Fitting the unknown coefficients of the short-range lattice interactions to empirical phase shifts can introduce significant uncertainties.

In this work we solve these problems by introducing a new set of short-range chiral EFT interactions on the lattice with a simpler decomposition into spin channels. The angular dependence of the relative separation between the two nucleons is prescribed by spherical harmonics, and the dependence on the nucleon spins is given by the spin-orbit Clebsch-Gordan coefficients. We first discuss the lattice Hamiltonian used in our lattice transfer matrix formalism. Next we discuss the numerical results. Finally, we present a summary and outlook.

\section{Lattice Hamiltonian and transfer matrix formalism}

We define the normal-ordered transfer matrix as

$$
M=: \exp \left[-H \alpha_{t}\right]:
$$

where the :: symbols denote normal ordering where the annihilation operators are on the right and creation operators are on the left. $\alpha_{t}=a_{t} / a$ is the ratio between the temporal lattice spacing and the spacial lattice spacing. We partition the lattice Hamiltonian $H$ into a free Hamiltonian, short-range interactions, and long-range interactions,

$$
H=H_{\text {free }}+V_{2 \mathrm{~N}}^{\text {short }}+V_{2 \mathrm{~N}}^{\text {long }} .
$$

For the free Hamiltonian we use an $O\left(a^{4}\right)$-improved action of the form [9],

$$
\begin{aligned}
H_{\text {free }}= & \frac{49}{12 m} \sum_{\mathbf{n}} a^{\dagger}(\mathbf{n}) a(\mathbf{n})-\frac{3}{4 m} \sum_{\mathbf{n}, i} \sum_{\left.\mathbf{n}^{\prime} \mathbf{n}\right\rangle_{i}} a^{\dagger}\left(\mathbf{n}^{\prime}\right) a(\mathbf{n}) \\
& +\frac{3}{40 m} \sum_{\mathbf{n}, i} \sum_{\left\langle\left\langle\mathbf{n}^{\prime} \mathbf{n}\right\rangle\right\rangle_{i}} a^{\dagger}\left(\mathbf{n}^{\prime}\right) a(\mathbf{n})-\frac{1}{180 m} \sum_{\mathbf{n}, i} \sum_{\left\langle\left\langle\left\langle\mathbf{n}^{\prime} \mathbf{n}\right\rangle\right\rangle\right\rangle_{i}} a^{\dagger}\left(\mathbf{n}^{\prime}\right) a(\mathbf{n}) .
\end{aligned}
$$

\subsection{Short-range interactions}

At order $Q^{0}$ we have two short-range interaction operators, namely, the $S$-wave spin singlet,

$$
V_{0,{ }^{1} S_{0}}(\mathbf{n})=\sum_{I_{z}=-1,0,1}\left[O_{0,0,0,0,1, I_{z}}^{0, S_{N L}}(\mathbf{n})\right]^{\dagger} O_{0,0,0,0,1, I_{z}}^{0, S_{N L}}(\mathbf{n})
$$

and the $S$-wave spin triplet,

$$
V_{0,{ }^{3} S_{1}}(\mathbf{n})=\sum_{J_{z}=-1,0,1}\left[O_{1,0,1, J_{z}, 0,0}^{0, s_{N L}}(\mathbf{n})\right]^{\dagger} O_{1,0,1, J_{z}, 0,0}^{0, s_{N L}}(\mathbf{n}) .
$$


At leading order we also include an SU(4)-invariant short-range operator $V_{0}$ with the form,

$$
V_{0}=\frac{C_{0}}{2}: \sum_{\mathbf{n}^{\prime}, \mathbf{n}, \mathbf{n}^{\prime \prime}} \sum_{i^{\prime}, j^{\prime}} a_{i^{\prime}, j^{\prime}}^{s_{\mathrm{NL}}^{\dagger}}\left(\mathbf{n}^{\prime}\right) a_{i^{\prime}, j^{\prime}}^{s_{\mathrm{NL}}}\left(\mathbf{n}^{\prime}\right) f_{s_{\mathrm{L}}}\left(\mathbf{n}^{\prime}-\mathbf{n}\right) f_{s_{\mathrm{L}}}\left(\mathbf{n}-\mathbf{n}^{\prime \prime}\right) \sum_{i^{\prime \prime}, j^{\prime \prime}} a_{i^{\prime \prime}, j^{\prime \prime}}^{s_{\mathrm{NL}}^{\dagger}}\left(\mathbf{n}^{\prime \prime}\right) a_{i^{\prime \prime}, j^{\prime \prime}}^{s_{\mathrm{L}}}\left(\mathbf{n}^{\prime \prime}\right):
$$

where $f_{s_{\mathrm{L}}}(\mathbf{n})=1$ for $|\mathbf{n}|=0, s_{L}$ for $|\mathbf{n}|=1$, and 0 otherwise.

At order $Q^{2}$ there are seven more operators, and at order $Q^{4}$ there are 15 more operators which are reduced to 12 after the on-shell constraint is taken into account. The specific expressions of these higher-order operators can be found in [10].

\subsection{Long-range interactions}

In Chiral EFT, the sources of the long-range interaction arise from the one-pion exchange potential with the form,

$$
V_{\mathrm{OPE}}=-\frac{g_{A}^{2}}{8 F_{\pi}^{2}} \sum_{\mathbf{n}^{\prime}, \mathbf{n}, S^{\prime}, S, I}: \rho_{S^{\prime}, I}\left(\mathbf{n}^{\prime}\right) f_{S^{\prime} S}\left(\mathbf{n}^{\prime}-\mathbf{n}\right) \rho_{S, I}(\mathbf{n}):
$$

and the two-pion exchange potential,

$$
V_{\mathrm{TPEP}}=V_{\mathrm{TPEP}}^{Q^{2}}+V_{\mathrm{TPEP}}^{Q^{3}}+V_{\mathrm{TPEP}}^{Q^{4}}
$$

\section{Results for the neutron-proton scattering}

We determine the LECs by reproducing the neutron-proton scattering phase shifts and mixing angles of the Nijmegen partial wave analysis (NPWA) [11]. The results are presented in Fig. (1). Good convergence is observed with increasing chiral order. With the full NN interactions up to order $O\left(Q^{4}\right)$, the calculation using $a=0.99 \mathrm{fm}$ can describe the $S, P$, and $D$ waves with a good accuracy over the whole momentum range, $0<p_{\text {rel }}<250 \mathrm{MeV}$.

At distance $r$ beyond the range of the interaction, the radial wave function for the deuteron in the ${ }^{3} S_{1}$ channel behaves as,

$$
u(r)=A_{S} e^{-\gamma r}
$$

where $A_{S}$ is the $S$-wave asymptotic normalization coefficient. In the ${ }^{3} D_{1}$ channel, the radial wave function behaves as

$$
w(r)=\eta A_{S}\left[1+\frac{3}{\gamma r}+\frac{3}{(\gamma r)^{2}}\right] e^{-\gamma r} .
$$

In Fig. (2), we show the radial wave functions of the deuteron. From the plots, one can see clearly that when the neutron and proton are well separated the $S$ and $D$ waves behave as the asymptotic forms in Eq. (3.1) and (3.2) respectively. The numerical values for $A_{S}$ and $\eta$ are shown in Table 1.

Using the radial wave functions, we can also compute the root-mean-square radius of the deuteron,

$$
r_{d}=\frac{1}{2}\left[\sum \delta r r^{2}\left(u^{2}(r)+w^{2}(r)\right)\right]^{1 / 2},
$$



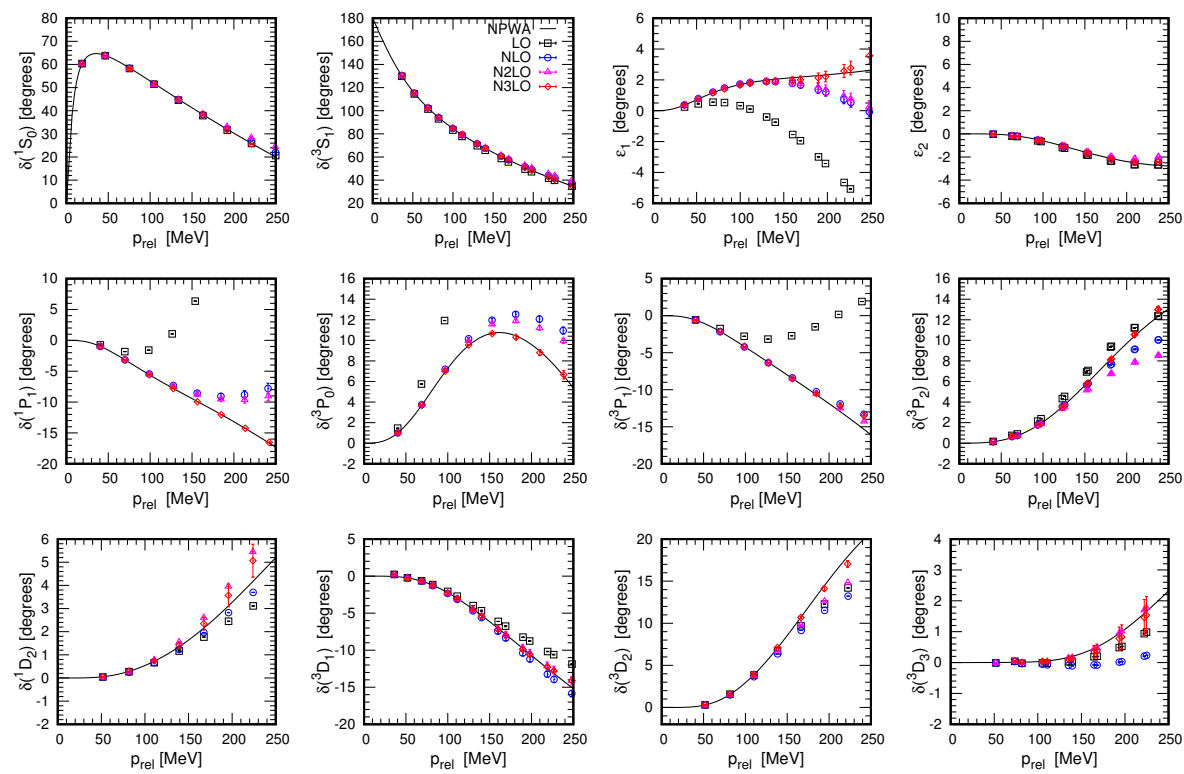

Figure 1: (Color online) Neutron-proton scattering phase shifts and mixing angles versus relative momenta.

\begin{tabular}{c|cccrr}
\hline \hline & LO & NLO & $\mathrm{N}^{2} \mathrm{LO}$ & \multicolumn{1}{c}{$\mathrm{N}^{3} \mathrm{LO}$} & \multicolumn{1}{c}{ Empirical } \\
\hline$E_{d}(\mathrm{MeV})$ & $2.2246 \pm 0.0002$ & $2.224575 \pm 0.000016$ & $2.224575 \pm 0.000025$ & $2.224575 \pm 0.000011$ & $2.224575(9)[12]$ \\
$A_{s}\left(\mathrm{fm}^{-1 / 2}\right)$ & $0.8662 \pm 0.0007$ & $0.8772 \pm 0.0003$ & $0.8777 \pm 0.0004$ & $0.8785 \pm 0.0004$ & $0.8846(9)[13]$ \\
$\eta$ & $0.0212 \pm 0.0000$ & $0.0258 \pm 0.0001$ & $0.0257 \pm 0.0002$ & $0.0254 \pm 0.0001$ & $0.0256(4)[14]$ \\
$Q_{d}\left(\mathrm{fm}^{2}\right)$ & $0.2134 \pm 0.00000$ & $0.2641 \pm 0.0016$ & $0.2623 \pm 0.0023$ & $0.2597 \pm 0.0013$ & $0.2859(3)[15]$ \\
$r_{d}(\mathrm{fm})$ & $1.9660 \pm 0.0001$ & $1.9548 \pm 0.0005$ & $1.9555 \pm 0.0008$ & $1.9545 \pm 0.0005$ & $1.97535(85)[16]$ \\
$a_{3} S_{1}$ & $5.461 \pm 0.000$ & $5.415 \pm 0.001$ & $5.421 \pm 0.002$ & $5.417 \pm 0.001$ & $5.424(4)[17]$ \\
$r_{3} S_{1}$ & $1.831 \pm 0.0003$ & $1.759 \pm 0.002$ & $1.760 \pm 0.003$ & $1.758 \pm 0.002$ & $1.759(5)[17]$ \\
$a_{1} S_{0}$ & $-23.8 \pm 0.1$ & $-23.69 \pm 0.05$ & $-23.8 \pm 0.2$ & $-23.678 \pm 0.038$ & $-23.748(10)[17]$ \\
$r_{1} S_{0}$ & $2.666 \pm 0.001$ & $2.647 \pm 0.003$ & $2.69 \pm 0.02$ & $2.647 \pm 0.004$ & $2.75(5)[17]$ \\
$P_{D}(\%)$ & 1.92 & 3.48 & 3.41 & 3.36 & \\
\hline \hline
\end{tabular}

Table 1: Deuteron properties and $S$-wave parameters calculated with the full NN interaction up to chiral order $O\left(Q^{4}\right)$ using $a=0.99 \mathrm{fm}$. Error bars we list here indicate uncertainties from the fitting procedure only.
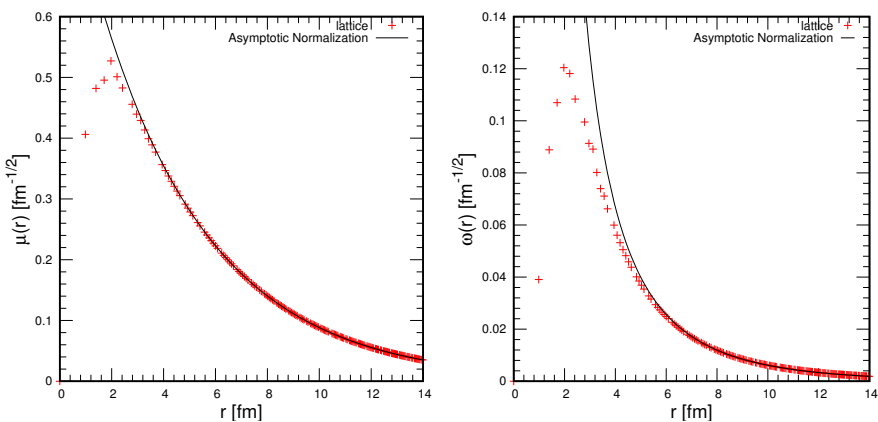

Figure 2: (Color online) Radial wave functions of the deuteron Left: $S$ wave. Right: $D$ wave. 
and the quadrupole moment,

$$
Q_{d}=\frac{1}{20} \sum \delta r r^{2} w(r)[\sqrt{8} u(r)-w(r)] .
$$

Additionally, the $S$-wave effective range parameters at very low energies can be extracted from

$$
p \cot (\boldsymbol{\delta})=-\frac{1}{a}+\frac{1}{2} r p^{2}+O\left(p^{4}\right)
$$

In Table 1, we present the properties of the deuteron and $S$-wave parameters. It is clear that the deuteron properties can be reproduced accurately. There are, however, still some small systematic discrepancies that suggest additional corrections are needed.

\section{Theoretical uncertainties}

It is necessary also to address the convergence of the effective field theory expansion on the lattice and their associated systematic errors. We follow the prescription in Refs. [18, 19] where the theoretical uncertainty for some observable $X(p)$ at order $\mathrm{N}^{m} \mathrm{LO}$ and momentum $p$ is given by

$\Delta X^{\mathrm{N}^{m} \mathrm{LO}}(p)=\max \left(Q^{m+2}\left|X^{\mathrm{LO}}(p)\right|, Q^{m}\left|X^{\mathrm{LO}}(p)-X^{\mathrm{NLO}}(p)\right|, \cdots, Q^{1}\left|X^{\mathrm{N}^{m-1} \mathrm{LO}}(p)-X^{\mathrm{N}^{m} \mathrm{LO}}(p)\right|\right)$.

In Fig. (3), we show the estimated theoretical uncertainties for the neutron-proton scattering phase shifts and mixing angles. With only a few exceptions, the error bands for each order generally overlap with each other and cover the empirical phase shifts, which provides a promising sign of convergence of the chiral effective field theory expansion on the lattice.

\section{Spectrum of the light and medium-mass nuclei}

With the new lattice action, we start calculating the binding energy of the light and mediummass nuclei. In Fig. (4) we show some preliminary results[20]. It it clear that the binding energy of the light nuclei are pretty accurate with the new interaction, although there are some discrepancy for the medium-mass nuclei. We should emphasize that in these calculations only the two-body interaction is applied. Hopefully, these discrepancy can be fixed by including the three-body interaction.

\section{Summary and outlook}

We have proposed a new lattice formulation of the chiral NN force which is easily decomposed into partial waves. The new lattice operators work as projection operators, which only survive in particular channels. This advantage greatly simplifies the fitting procedure. Instead of fitting the phase shifts and mixing angles for all the channels simultaneously, only one uncoupled channel or two coupled channels are needed to be computed for each calculation.

We have also studied the properties of the deuteron wave function and the $S$-wave effective range parameters. The numerical values are very close to the empirical values, which indicates that the current version of NN interactions is quite accurate, and a very significant improvement over 

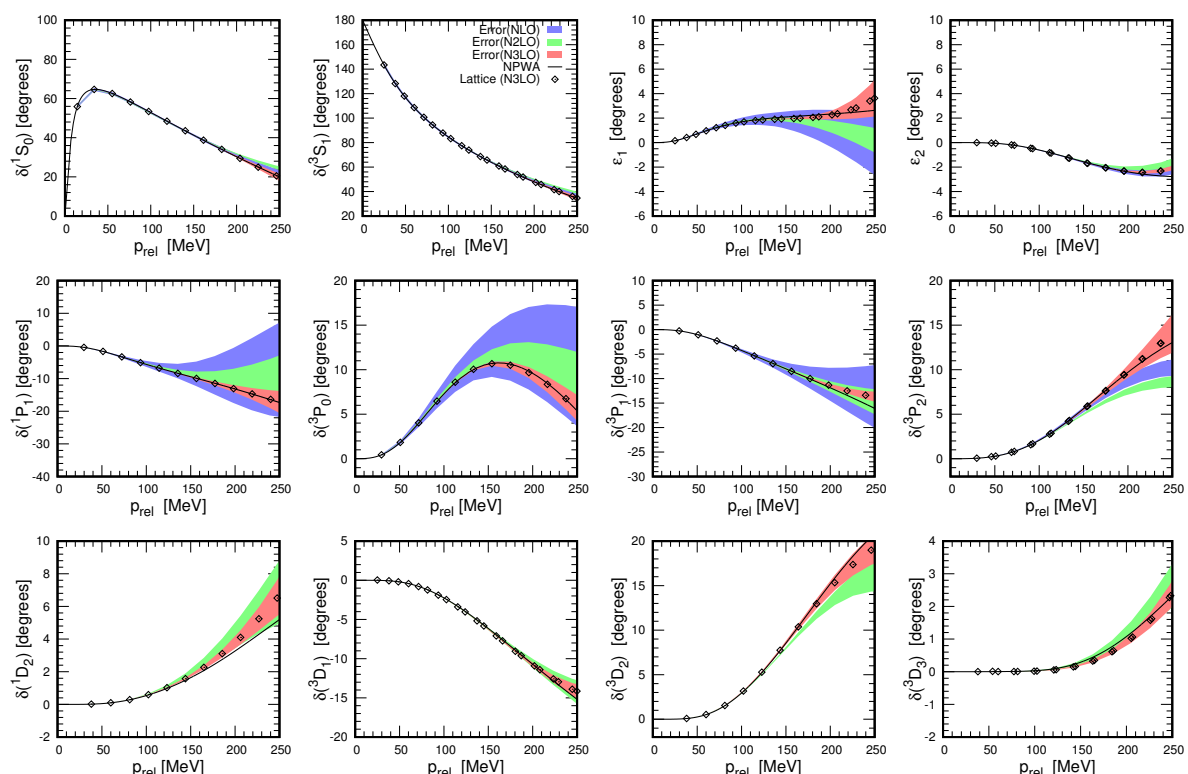

Figure 3: (Color online) Theoretical error bands for the neutron-proton scattering phase shifts and mixing angles versus the relative momenta. Blue, green, and red bands signify the estimated uncertainties at NLO, $\mathrm{N}^{2} \mathrm{LO}$ and $\mathrm{N}^{3} \mathrm{LO}$ respectively. The black solid line and diamonds denote the phase shift or mixing angle from the Nijmegen partial-wave analysis (NPWA) and lattice calculation at $\mathrm{N}^{3} \mathrm{LO}$, respectively.

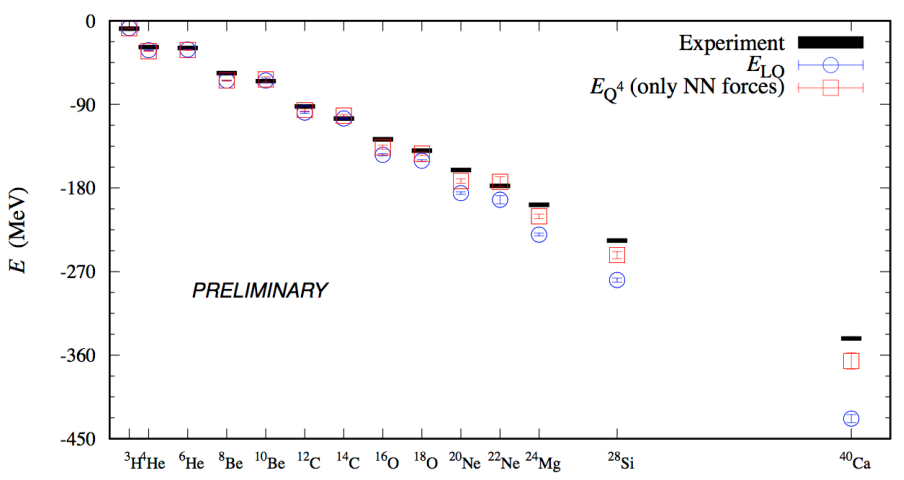

Figure 4: Spectrum of the light and medium-mass nuclei. In the calculations only the two-body interactions are applied.

previous lattice studies. Some small discrepancies remain, but these may well be fixed in studies that reach a higher order in the chiral effective field theory expansion.

In summary, the new lattice interactions are far more efficient and accurate in reproducing physical data than previous lattice interactions. We have begun studying the properties of light and medium-mass nuclei using these interactions, and the results are promising.

\section{References}

[1] E. Epelbaum, H. Krebs, D. Lee and U.-G. Meißner, Ab initio calculation of the Hoyle state, Phys. Rev. Lett. 106 (2011) 192501 [1101.2547]. 
[2] E. Epelbaum, H. Krebs, T. Lähde, D. Lee and U.-G. Meißner, Structure and rotations of the Hoyle state, Phys. Rev. Lett. 109 (2012) 252501 [1208.1328].

[3] E. Epelbaum, H. Krebs, T. A. Lähde, D. Lee, U.-G. Meißner and G. Rupak, Ab initio calculation of the spectrum and structure of ${ }^{16}$ O, Phys. Rev. Lett. 112 (2014) 102501 [1312. 7703 ].

[4] S. Elhatisari, D. Lee, G. Rupak, E. Epelbaum, H. Krebs, T. A. Lähde et al., Ab initio alpha-alpha scattering, Nature 528 (2015) 111 [1506.03513].

[5] S. Elhatisari et al., Nuclear binding near a quantum phase transition, Phys. Rev. Lett. 117 (2016) 132501 [1602.04539].

[6] S. Elhatisari, E. Epelbaum, H. Krebs, T. A. Lähde, D. Lee, N. Li et al., Ab initio Calculations of the Isotopic Dependence of Nuclear Clustering, Phys. Rev. Lett. 119 (2017) 222505 [1702. 05177 ].

[7] J. M. Alarcón, D. Du, N. Klein, T. A. Lähde, D. Lee, N. Li et al., Neutron-proton scattering at next-to-next-to-leading order in Nuclear Lattice Effective Field Theory, Eur. Phys. J. A53 (2017) 83 [1702.05319].

[8] N. Klein, S. Elhatisari, T. A. Lähde, D. Lee and U.-G. Meißner, The Tjon Band in Nuclear Lattice Effective Field Theory, Eur. Phys. J. A54 (2018) 121 [1803.04231].

[9] D. Lee, Lattice simulations for few-and many-body systems, Prog. Part. Nucl. Phys. 63 (2009) 117 [0804.3501].

[10] N. Li, S. Elhatisari, E. Epelbaum, D. Lee, B.-N. Lu and U.-G. Meißner, Neutron-proton scattering with lattice chiral effective field theory at next-to-next-to-next-to-leading order, Phys. Rev. C98 (2018) $044002[1806.07994]$.

[11] V. G. J. Stoks, R. A. M. Klomp, M. C. M. Rentmeester and J. J. de Swart, Partial wave analaysis of all nucleon-nucleon scattering data below 350-MeV, Phys. Rev. C48 (1993) 792.

[12] C. Van Der Leun and C. Alderliesten, The deuteron binding energy, Nucl. Phys. A380 (1982) 261.

[13] T. E. O. Ericson and M. Rosa-Clot, The Deuteron Asymptotic D State as a Probe of the Nucleon-nucleon Force, Nucl. Phys. A405 (1983) 497.

[14] N. L. Rodning and L. D. Knutson, Asymptotic D-state to S-state ratio of the deuteron, Phys. Rev. C41 (1990) 898.

[15] D. M. Bishop and L. M. Cheung, Quadrupole moment of the deuteron from a precise calculation of the electric field gradient in D-2, Phys. Rev. A20 (1979) 381.

[16] A. Huber, T. Udem, B. Gross, J. Reichert, M. Kourogi, K. Pachucki et al., Hydrogen-Deuterium S-1S-2 Isotope Shift and the Structure of the Deuteron, Phys. Rev. Lett. 80 (1998) 468.

[17] O. Dumbrajs, R. Koch, H. Pilkuhn, G. c. Oades, H. Behrens, J. j. De Swart et al., Compilation of Coupling Constants and Low-Energy Parameters. 1982 Edition, Nucl. Phys. B216 (1983) 277.

[18] E. Epelbaum, H. Krebs and U.-G. Meißner, Improved chiral nucleon-nucleon potential up to next-to-next-to-next-to-leading order, Eur. Phys. J. A51 (2015) 53 [1412.0142].

[19] E. Epelbaum, H. Krebs and U.-G. Meiß§ner, Precision nucleon-nucleon potential at fifth order in the chiral expansion, Phys. Rev. Lett. 115 (2015) 122301 [1412 . 4623].

[20] S. Elhatisari et al., work in process. 